\title{
Modelo de cultura organizacional innovadora en caficultores del departamento del Cesar, Colombia
}

\section{Model for innovative organizational culture in coffee growers in Cesar State, Colombia}

\section{Vanessa Pertuz-Peralta ${ }^{1}$ Adith Bismarck Pérez-Orozco ${ }^{2}$}

Recibido: julio 12 de 2015 Aceptado: diciembre 28 de 2015

\section{Resumen}

El objetivo del artículo es proponer un modelo para el desarrollo de una cultura organizacional innovadora en el sector empresarial a partir del análisis de la Asociación de Productores Orgánicos Arhuacos y Campesinos de la Sierra Nevada de Santa Marta, Asoprocasines. El presente estudio se desarrolla desde el enfoque cualitativo, mediante la tipología de estudio de caso. Los datos se obtuvieron de la observación directa, mediante una matriz de análisis. El modelo propuesto integra los aspectos determinantes en la generación de una cultura innovadora, considerando la revisión de la literatura al respecto. El modelo contempla cinco pasos que se retroalimentan: articulación de la innovación a la estrategia competitiva, desarrollo de competencias en el personal, retroalimentación con los clientes, planificación y seguimiento de estrategias de innovación y seguimiento a las acciones y estrategias. Adicionalmente, se incluyen dos elementos dinamizadores de este proceso: la adopción de la cultura de cambio y la interacción de la empresa con el ambiente externo. Asimismo, el modelo se apoya en los pasos para la gestión de la innovación planteados por la fundación COTEC en 2004.

Palabras clave: cultura organizacional, innovación, cultura innovadora, modelo de cultura innovadora.

\section{Abstract}

This paper aims to propose a new model for the development of an organizational innovative culture in the business sector. The model is inspired by the analysis of the Asociación de Productores Orgánicos Arhuacos y Campesinos de la Sierra Nevada de Santa Marta, Asoprocasines. This study is developed based on the qualitative approach, and it is validated by a case study. The data are recollected by direct observation, using an analysis matrix. The proposed model integrates the determinants factors in the generation of an innovative culture, based on the review of the literature. This model defines five steps: joint innovation to competitive strategy, developing skills in staff, customer feedback, planning and monitoring of innovation strategies, and monitoring the actions and strategies. The culture of change and the interaction of the company with the external environment are discussed. Additionally, two driving forces of this process are included, which are the adoption of culture of change and the interaction between company and external environment. Also, the model relies on the steps for managing innovation planed by the COTEC Foundation in 2004.

Keywords: organizational culture, innovation, innovative culture, innovative culture model.

\footnotetext{
1 Ingeniera Industrial, Magíster en Gerencia de Proyectos de Investigación y Desarrollo, Universidad de Santander, Colombia. E-mail: vanesapertuz@hotmail.com

2 Ingeniero de Sistemas, Magíster en Ingeniería Electrónica, Universidad de Santander, Colombia. E-mail: adithperez@unicesar.edu.co
} 


\section{Introducción}

El clima competitivo en el que se desarrollan las organizaciones en la actualidad requiere el desarrollo de innovaciones, con el objeto de consolidar ventajas competitivas sostenibles. La innovación implica el desarrollo de capacidades organizacionales y humanas, asociadas con la apertura al cambio, la comunicación efectiva, el trabajo en equipo, la aceptación del fracaso, la orientación humana y la creatividad; elementos que en su conjunto responden al tipo de cultura organizacional. Así, la cultura corresponde a la plataforma habilitante para los procesos de innovación a nivel organizacional.

En la literatura se evidencia el reconocimiento de la innovación como una fuente de ventajas competitivas y el efecto positivo de la cultura organizacional en el proceso innovador (Naranjo-Valencia, Jiménez, \& Sanz-Valle, 2012), (Naranjo-Valencia \& Calderón-Hernández, 2015). No obstante, los trabajos empíricos sobre la relación entre la cultura organizacional y la innovación son muy escasos; y "abordan las variables de la cultura de forma aislada y no de forma holística a través del estudio del efecto de los distintos arquetipos de cultura sobre la innovación" (Naranjo et al., 2012, p. 63). Adicionalmente, los autores en mención establecen que, pese a que los trabajos empíricos que analizan la relación entre innovación y cultura organizacional son heterogéneos tanto en las muestras empleadas como en el concepto de las medidas de cultura e innovación, en general evidencian que la cultura es un determinante de la innovación.

El objetivo del presente trabajo es el de proponer un modelo para el desarrollo de una cultura organizacional innovadora en el sector empresarial, a partir del análisis de la Asociación de Productores Orgánicos Arhuacos y Campesinos de la Sierra Nevada de Santa Marta "Asoprocasines". El estudio se desarrolló desde el enfoque cualitativo, mediante la tipología de estudio de caso. El modelo propuesto integra los aspectos determinantes en la generación de una cultura innovadora, consi- derando la revisión de la literatura al respecto. A continuación se incluye la conceptualización de cultura innovadora y se describe la metodología utilizada en la investigación. Asimismo, se presentan los resultados del estudio asociados con la caracterización de la cultura organizacional de la empresa analizada y el modelo propuesto para el desarrollo de una cultura organizacional. Finalmente, se enuncian las conclusiones.

\section{Marco teórico y metodología}

\subsection{Cultura organizacional e innovación}

De acuerdo con Rodríguez, Hoyos, Izaguirre y Vicente (2011), la cultura organizacional se concibe como un conjunto de valores y creencias profundamente arraigadas, compartidas por los miembros de la empresa, que guían su forma de relacionarse entre sí y con el exterior. Ayala (2014) establece que la cultura organizacional corresponde a la forma de interacción de los individuos de una organización, los cuales buscan de acuerdo a sus necesidades el logro de objetivos individuales, lo que lleva al cumplimiento de objetivos comunes con otros individuos y permite alcanzar los objetivos de la organización. Desde este ámbito, la cultura organizacional es la base a través de la cual se articulan los procesos y miembros de la organización, con el objeto de generar ventajas competitivas sostenibles.

Frente al concepto de innovación, Schumpeter (1934), quien fue el primero en destacar la importancia de los fenómenos tecnológicos en el crecimiento económico, definió la innovación en un sentido más general que el de las específicamente tecnológicas. Según Pavon y Goodman (1981), la innovación corresponde al conjunto de actividades inscritas en un determinado período de tiempo y lugar, que conducen a la introducción con éxito, por primera vez, de una idea en forma de nuevos o mejores productos, servicios o técnicas de gestión y organización. La conceptualización aportada por Escorsa y Valls (2003) establece que 
la innovación es la explotación con éxito de nuevas ideas: innovación = invento + explotación.

Según el Manual de Frascati de la OCDE (2003), el concepto de innovación corresponde a la transformación de una idea en un producto o servicio comercializable, un procedimiento de fabricación o distribución operativo; o un método de proporcionar un servicio social, nuevo o mejorado. El manual de Oslo define la innovación como "la implementación de un producto, o proceso, nuevo o significativamente mejorado, un nuevo método de comercialización, o un nuevo método organizacional en las prácticas de negocio, la organización del lugar de trabajo o las relaciones externas" (OECD \& Eurostat, 2005, p.56).

Así, la cultura innovadora se define como la conducta desarrollada por algunas organizaciones orientadas por el impulso a ideas creativas de sus miembros, estimulados por un ambiente de trabajo caracterizado por una alta motivación al logro, asignación de recursos para darle soporte a los procesos y estímulos a las habilidades demostradas por las personas capaces de producir ideas originales y exitosas, con el fin de satisfacer las demandas de conocimiento en un ambiente competitivo y globalizado (Ramos-Parra, 2006).

De acuerdo con Naranjo-Valencia y Calderón-Hernández (2015), en la actualidad la cultura organizacional es considerada como uno de los factores que más puede estimular la conducta innovadora en los colaboradores de una organización. No obstante, según los autores en referencia, la cultura organizacional no siempre puede favorecer la innovación, en algunos casos, pueden dificultarla dependiendo de los rasgos o valores que fomente, y en consecuencia, de los comportamientos que estimule en los colaboradores. Asimismo, el horizonte del proceso de creación de una cultura innovadora se orienta a generar en la organización la capacidad proactiva de explorar nuevas oportunidades y desarrollar esfuerzos innovadores capaces de exceder las expectativas de los clientes, para así permitir el logro de ventajas competitivas (Hult, Hurley, \& Knight, 2004).

2.2 Bases conceptuales del modelo de cultura innovadora

En esta sección se presenta la revisión de la literatura considerada para la estructuración de cada uno de los componentes del modelo de cultura de innovación aquí propuesto.

Articulación de la innovación a la estrategia competitiva

La innovación exige una lectura constante del entorno y de las capacidades organizacionales, lo cual implica que la cultura innovadora debe favorecer la orientación externa como un medio. Así, la innovación no se puede desarrollar sin comprender adecuadamente el comportamiento del mercado y su posible respuesta (Deshpandé $\&$ Farley, 2004). Igualmente, la integración de la innovación como parte transversal de la estrategia, supone la interacción de los recursos organizacionales y del talento humano como fuerza motora, a través del empoderamiento y participación en el proceso de innovación.

\section{Desarrollo de competencias en el personal}

Según De Brentani y Ragot (1996), la superación de las barreras internas que surgen en el personal para la implementación de culturas organizacionales innovadoras se logra mediante el fortalecimiento de aspectos como: el compromiso de los colaboradores, que genera un alto desempeño creativo y favorece su motivación; así mismo, la comunicación efectiva entre los miembros, considerando que en una cultura innovadora deben valorarse las aportaciones de los colaboradores en los procesos de creación de nuevos productos, contribuyendo con la motivación y al refuerzo del compromiso del personal (Ottenbacher, Gnoth, \& Jones, 2006). 
Una organización innovadora no solo exige destinar recursos a la investigación y desarrollo interna/ externa, sino que además, requiere una cultura organizativa que constantemente incite a sus miembros a que se esfuercen por innovar y a que en la empresa exista un clima que estimule la creatividad (Rodríguez, Araujo, Hagemeister, Landeta, Larrauri, \& Ranguelov, 2006). En el mismo sentido, De Brentani y Ragot (1996) indican que la verdadera cultura innovadora es la que consigue, junto con el compromiso de los directivos de la empresa, que los colaboradores participen de forma activa en los proyectos de desarrollo y lanzamiento de nuevos bienes y servicios.

Igualmente, Naranjo-Valencia y Calderón-Hernández (2015) plantean las características de las culturas organizacionales que favorecen la innovación: creatividad, iniciativa, espíritu emprendedor; libertad/autonomía; ausencia del riesgo; trabajo en equipo; suficiencia de recursos; orientación estratégica hacia el cliente; toma de decisiones; participación de los trabajadores; aprendizaje autónomo y flexibilidad.

\section{Retroalimentación con clientes}

La fuente más importante para la innovación son los clientes, seguidos del personal de ventas y los empleados; así, mientras más dinámica e innovadora es la empresa, tiene mayor relevancia la función de los consumidores en la innovación (Sundbo, 1997; Sundbo \& Gallouj, 2000). Favorecer la participación de los clientes en el proceso innovador favorece la retroalimentación frente a la calidad y la eficacia, mediante procesos de desarrollo más rápidos y pertinentes con las exigencias del mercado, dinámica que garantiza la creación de ventajas competitivas sostenibles. Las empresas con una fuerte predisposición a innovar estarán más dispuestas a contar con la colaboración de sus clientes no solo como fuentes de ideas, sino incluso como co-creadores, considerando que la aportación de los clientes juega un papel esencial en el desarrollo de innovaciones (Hennestad, 1999).
Producto del desarrollo de las competencias del personal y la retroalimentación con los clientes, se genera la cultura co-creadora propuesta por Ballantyne, Varey, Frow y Payne (2008), en el cual se destaca la importancia de los empleados y de los clientes como co-creadores en la innovación (Alam, 2005; Ottenbacher, et al., 2006). Puntualmente, la cultura co-creadora genera ventajas tales como: la obtención de información de los colaboradores que constituyen una fuente esencial de innovación (Dewitt \& Brady, 2003) y el aumento de la predisposición a innovar, la cual, junto con una actitud favorable hacia la incorporación de nuevas ideas, fomenta el desarrollo de las competencias claves en la innovación (Hurley \& Hult, 1998). La cultura innovadora debe promover la participación de todos los miembros de la organización en los procesos de innovación.

Planificación y documentación de estrategias de innovación

La generación de una cultura innovadora incluye la planificación y documentación de las estrategias de innovación, lo cual es relevante para obtener resultados sostenibles en la organización; esto se debe a que favorece el proceso de monitoreo de las metas planteadas, generando la retroalimentación y el aprendizaje producto del ejercicio de planificación y seguimiento de las estrategias de innovación, articuladas con la estrategia global.

\section{Seguimiento a las estrategias y acciones}

La generación de una cultura innovadora implica los procesos de seguimiento a las estrategias y acciones de innovación, con el objeto de identificar las debilidades, las fortalezas de los procesos organizacionales y las oportunidades del entorno para generar ventajas competitivas en la organización.

\section{Adopción de la cultura de cambio}

La literatura sugiere que aquellas organizaciones que cuentan con una mayor cultura innovado- 
ra, o con una mayor disposición para aceptar los riesgos inherentes a los procesos de innovación y para emprender los cambios técnicos y organizativos precisos para su desarrollo, obtienen mejores resultados empresariales (Griffin, 1997; Hult et al., 2004; Menguc \& Auh, 2006). Desde esta perspectiva, la cultura innovadora se corresponde con un componente clave del proceso competitivo.

\section{Interacción con el ambiente externo}

La organización es concebida como un elemento de un sistema, donde influyen diferentes grupos de interés, por lo que para el desarrollo y el fomento de la innovación, es necesario conjugar una serie de cualidades personales innatas o adquiridas como: la creatividad, la flexibilidad y el conocimiento (Rodríguez et al., 2011), con un entorno o ecosistema más amplio integrado por organizaciones, agentes sociales, instituciones, sistema educativo, redes; en el que se fomente y se pre- mie la innovación, de forma que esta sea percibida como un objetivo beneficioso para la sociedad (Future Trends Forum, 2007).

\section{Modelo de la fundación COTEC}

De acuerdo con la Fundación para la Innovación tecnológica, COTEC (2004), la gestión de la innovación se fundamenta en cinco etapas interrelacionadas: focalizar, capacitarse, implantar, vigilar y aprender.

\subsection{Metodología}

La presente investigación propone un modelo para la generación de la cultura innovadora en la empresa Asoprocasines, Asociación de productores Orgánicos Arahuacos y Campesinos de la Sierra Nevada de Santa Marta, cuyas características se presentan en la tabla 1.

\begin{tabular}{ll}
\hline Actividad comercial: & $\begin{array}{l}\text { Producción y comercialización de café orgánico, marca "Café } \\
\text { Nacer" }\end{array}$ \\
$\begin{array}{l}\text { Clasificación jurídica: } \\
\text { Localización: }\end{array}$ & $\begin{array}{l}\text { Asociación } \\
\text { Pueblo Bello, Cesar } \\
\text { Tiempo de constitución: }\end{array}$ \\
$\begin{array}{l}17 \text { años } \\
\text { No de colaboradores: }\end{array}$ & 5 (Directos) \\
USDA Organic; CO-BIO-141 Agricultura de Colombia; FAIR- \\
Certificaciones: & $\begin{array}{l}\text { TRADE Comercio Justo; Código de barras; OKO Garantie BCS } \\
\text { (ente certificador) }\end{array}$ \\
Mercado objetivo: & Mercado nacional e internacional \\
\hline
\end{tabular}

Tabla 1. Caracterización de la empresa Asoprocasines.

El trabajo se desarrolla desde un enfoque cualitativo, el cual "se enfoca en comprender y profundizar los fenómenos, explorándolos desde la perspectiva de los participantes en un ambiente natural y en relación con el contexto" (Hernández, Fernández, \& Baptista, 2010, p. 364). El proyecto metodológicamente se ubica en un estudio de caso, el cual "es apropiado en situaciones en las que se desea estudiar intensivamente características básicas, la situación actual e interacciones con el medio de una o unas pocas unidades tales como individuos, grupos, instituciones o comunidades" (Tamayo \& Tamayo, 2011, p. 51).

El presente trabajo se estructura bajo un diseño de tipo no experimental, transeccional y de campo (Hernández et al., 2010). Los datos se obtienen a través de la técnica de la observación directa utilizando el diseño de una matriz de análisis (Finol \& Nava, 1999). La matriz compara las características 
referenciadas en la revisión de literatura con las observadas en la empresa; y la ficha técnica de los artículos considerados en la revisión bibliográfica (Tamayo \& Tamayo, 2011). La investigación utiliza esta aproximación debido a que "La literatura empírica, centrada en estudiar la relación entre diferentes tipos de cultura organizativa y la innovación, hasta el momento es muy escasa" (Naranjo, et al., 2012, p. 66).

La unidad de análisis es la Asociación de Productores Orgánicos Arhuacos y Campesinos de la Sierra Nevada de Santa Marta, Asoprocasines; una organización de pequeños Productores Orgánicos Indígenas y Arhuacos de la Sierra Nevada de Santa Marta, conformada por 236 asociados, 40\% campesinos y $60 \%$ indígenas, con capacidad de producción de 5000 sacos (350 TM) de café verde, comercializado internacionalmente bajo el nombre de Café Flo Orgánico Nacer, a través de la Federación Nacional de Cafeteros, a América del Norte, Europa y Asia (Asoprocasines, 2011). En síntesis, la metodología se centra en la revisión bibliográfica de los conceptos de cultura organizacional innovadora y su aplicación a un contexto específico, producto de un estudio de caso en Asoprocasines, para determinar los factores y características que servirían a la generación de una cultura innovadora.

\section{Resultados y discusión}

3.1 Análisis diagnóstico de la cultura organizacional

En esta sección se expone la caracterización de la cultura organizacional de la empresa Asoprocasines, objeto de estudio de la presente investigación, a partir de los aspectos teóricos contemplados en la revisión de literatura.

\section{Tipo de cultura organizacional}

Respecto a la tipología de cultura organizacional aportada por el modelo "Valores en competencia" de Cameron y Quinn (1999), se identifican cuatro tipos de cultura organizacional: racional, jerárquica, de grupo y de desarrollo. Al respecto, en el análisis diagnóstico se identifican los elementos correspondientes a cada uno de los tipos de cultura tratados en la literatura. En primer lugar se evidencia una cultura de grupo, debido a las relaciones interpersonales basadas en el afecto y la cercanía, motivado especialmente por el hecho de que la empresa surgió como iniciativa de un conjunto de familias para generar valor agregado en torno al café. Igualmente, se presentan características propias de una cultura de desarrollo, debido a la marcada orientación hacia el crecimiento.

La caracterización de la cultura racional y jerárquica en Asoprocasines, se fundamenta en su dependencia funcional con la institución pública que representa la institucionalidad gremial cafetera en Colombia, Federación Nacional de Cafeteros, así como en organismos privados que se constituyen como intermediarios en el proceso de exportación del café. Lo anterior se refleja en la cultura organizacional generando una fuerte orientación a la productividad y a la calidad del café, correspondiente a la organización de una cultura racional, así como la presencia de una cultura jerárquica, limitando la generación de escenarios de participación para la toma de decisiones.

En líneas generales, la caracterización de la cultura organizacional de Asoprocasines evidencia, en mayor medida, elementos correspondientes a la tipología de una cultura de grupo y racional. Cabe destacar que se tomó como referencia de la tipología de cultura organizacional el modelo propuesto por Cameron y Quinn (1999), debido a que corresponde a la tipología "más extendida y ampliamente utilizada en la literatura" (Naranjo-Valencia \& Calderón-Hernández, 2015, p. 225). 
Tipología de las organizaciones según su cultura tecnológica

Pirela, Rengifo, Arvanitis y Mercado (1991) identifican las siguientes tipologías de organizaciones según su cultura tecnológica: pasiva, reactiva y activa. Asoprocasines se ubica dentro de una cultura tecnológica reactiva, considerando los procesos de adaptación de la tecnología provenientes de la Federación Nacional de Cafeteros, la cual desarroIla herramientas y sistemas de información orientadas a la solución de las problemáticas asociadas al cultivo del café y la eficiencia operativa de las organizaciones. Así, dentro de la organización hay un creciente interés por realizar la adaptación y el aprovechamiento de este tipo de tecnologías, y en menor medida, en desarrollar herramientas tecnológicas que permitan potenciar los procesos.

\section{Elementos constitutivos de la cultura organizacional}

Arias y Heredia (2001) plantean los siguientes elementos como constitutivos de la cultura organizacional: relación de la organización con el entorno, naturaleza de la actividad humana, la realidad y la verdad, el tiempo, la naturaleza humana, las relaciones interpersonales, la homogeneidad y diversidad. En este sentido, la empresa ha consolidado una adecuada relación con su entorno, como parte del cumplimiento de las normas internacionales en las que está certificada, especialmente el sello Fairtrade, Comercio Justo, que identifica sus productos; sumado a un sólido compromiso con el medio ambiente y el mejoramiento de la calidad de las familias vinculadas. Desde el ámbito de la naturaleza humana, existe una alta participación de los miembros en la conducción de la empresa, mediante la ejecución de proyectos y el desarrollo de actividades complementarias al cumplimiento de las disposiciones misionales que rigen el ejercicio organizacional.

En cuanto al tiempo, el análisis de la cultura organizacional evidencia una orientación al presente con iniciativa al futuro. La organización ha logrado consolidarse y competir efectivamente en su segmento de mercado y consolidar una visión de futuro, apoyándose en alianzas estratégicas. En el ámbito de la naturaleza humana, se evidencia una actitud comprometida de los miembros hacia la organización, reflejadas en el desarrollo de relaciones interpersonales óptimas y en un liderazgo participativo.

Determinantes para el desarrollo de la cultura organizacional

Schein (1992) identifica como determinantes para el desarrollo de la cultura organizacional fuerte o débil, los siguientes aspectos: la creación de un sentido de historia, representados en la misión y visión organizacional, la generación de un sentido de unicidad, el fomento de un sentido de pertenencia y el incremento del intercambio interpersonal entre los miembros de la organización. La empresa analizada cuenta con una misión y visión consolidada entre sus miembros, elemento significativo en la creación del sentido de historia; se evidencia un fuerte desarrollo del sentido de la unicidad y pertenencia, en el marco de una interacción armónica entre los miembros.

Herramientas destinadas a impulsar cambios en la cultura organizacional

En referencia a las herramientas destinadas a impulsar cambios en la cultura organizacional, Gibson, Ivancevich y Donnelly (2002) establecen las siguientes: impulsar cambios en los comportamientos de las personas, destacar el mérito inherente al nuevo comportamiento y establecer mecanismos efectivos de comunicación interna, socialización de nuevos miembros, así como el despido de miembros que no se ajusten a la nueva cultura. Al respecto, el análisis diagnóstico evidencia que en Asoprocasines no se activan de manera sistemática los mecanismos de cambio con el objeto de favorecer la implementación de elementos estratégicos y cambios en la cultura organizacional. 
Este resultado obedece a la concepción de que la cultura organizacional corresponde a un elemento individualizado de la gestión de la empresa, excluyendo el enfoque integrador, dentro del cual la cultura se articula como la plataforma habilitante y necesaria para el fortalecimiento y la implementación de procesos organizacionales. Lo anterior pese a que el mercado objetivo de la organización es el segmento internacional, hecho que le plantea exigencias específicas y oportunidad en las respuestas frente a los cambios del entorno. En el mismo sentido, es importante fortalecer la capacidad de seguimiento a las estrategias planteadas, como un factor clave en la competitividad y en el desarrollo de la organización.

\section{Proceso de administración estratégica}

Según lo planteado por Hill y Jones (2001), el proceso de administración estratégica incluye los siguientes aspectos: la selección de la visión y misión de forma participativa, la elección de las principales metas corporativas, la realización del análisis FODA: fortaleza, oportunidad, debilidad y amenaza; esto se une a la selección de estrategias fundamentadas en las fortalezas de la organización para que corrijan sus debilidades con el fin de tomar ventaja de las oportunidades externas. Lo anterior se complementa con la implementación de la estrategia, el logro de los objetivos y la retroalimentación.

El análisis del proceso de administración estratégica muestra que en la organización se efectúan actividades relacionadas con este; sin embargo, estas corresponden a iniciativas aisladas, ausentes del enfoque de planificación, ejecución sistemática, verificación del cumplimiento y mejora continua, orientado a la mejora de los procesos organizacionales.

\section{Elementos relevantes en la cultura organizacional}

Ramos-Parra (2012) identifica como elementos relevantes en la cultura de las organizaciones los siguientes elementos: la comunicación fluida, la interacción de los miembros del equipo de trabajo, la atención al cliente interno y externo, las estrategias de participación, la toma de decisiones por consenso y acuerdo, la planificación estratégica más allá de lo operativo o cotidiano y la homogeneidad de las ideas en los grupos. El análisis diagnóstico indica que en Asoprocasines existe una comunicación fluida, una alta interacción de los miembros del equipo de trabajo y un proceso efectivo de atención al cliente interno y externo. Asimismo, se evidencia la marcada aplicación de las estrategias de participación para la toma de decisiones orientadas a la búsqueda de homogeneidad, así como la toma de decisiones por consenso y acuerdo relacionadas con actividades internas específicas de la organización, elementos que soportan los procesos organizacionales. No obstante, el seguimiento a la planeación se concentra en el cumplimiento de los procesos operativos relacionados con la producción, por lo que resulta fundamental establecer y monitorear la planeación de las diferentes estrategias incluidas dentro de la planeación organizacional.

Características de la cultura organizacional que fortalecen la competitividad

La literatura plantea una marcada influencia de la cultura organizacional sobre la competitividad de la organización; al respecto, Hitt, Ireland y Hoskisson (1999), identificaron siete características de la cultura organizacional que fortalecen la competitividad, a saber: la promoción del aprendizaje y del desarrollo humano, la distribución de los talentos y recursos entre las unidades organizacionales, la innovación, la visión de largo plazo, la calidad de los productos y servicios, la cooperación y los equipos de trabajo y valores éticos.

En relación con lo anterior, en Asoprocasines se identifican las siguientes características: promoción del aprendizaje y desarrollo, motivado por el desarrollo de proyectos en alianzas estratégicas, así como de procesos de transferencia de conocimiento como parte del proceso de dirección liderado por la Federación Nacional de Cafeteros. 
Igualmente, se demuestra una óptima distribución de los talentos y recursos entre las unidades organizacionales, ámbito en el cual tiene una importancia significativa el conocimiento empírico del proceso de cultivo del café. Sin embargo, se observa un bajo impulso innovador en los productos y en los procesos, que en general responden al cumplimiento de los parámetros exigidos, sin elementos diferenciadores desarrollados.

\section{Atenuantes para el éxito del modelo de cultura orga- nizacional}

Páramo (2001) establece como atenuantes para el éxito del modelo de cultura organizacional orientado al mercado, la existencia de un adecuado clima organizacional, representado en las estructu- ras, los sistemas, y los incentivos para implementar los valores y el espíritu empresarial, a través del liderazgo creativo. Considerando los atenuantes en referencia, es importante indicar que Asoprocasines cuenta con un clima organizacional efectivo para implementar los valores y las políticas de la organización, así como un marcado espíritu creativo a través del liderazgo.

\subsection{Modelo propuesto}

En la figura 1 se presenta la estructura del modelo propuesto para la generación de una cultura innovadora; luego se hace la descripción de cada componente, basada en los referentes teóricos considerados en la revisión de la literatura.

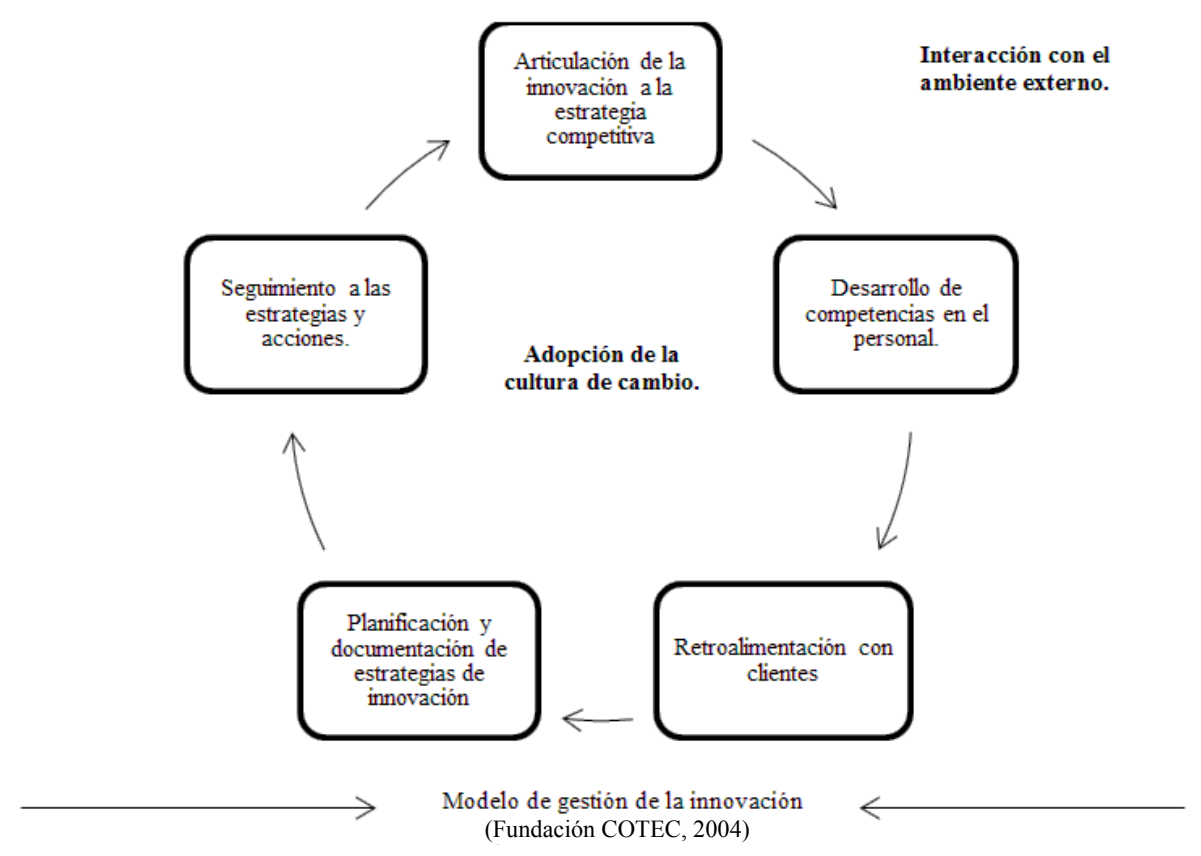

Figura 1. Estructura del modelo propuesto para la generación de una cultura innovadora.

El modelo propuesto para el desarrollo de una cultura innovadora se sustenta en cinco componentes que se retroalimentan: el primero, se asocia con la articulación de la innovación y la responsabilidad social empresarial a la estrategia competitiva de la organización. Este componente se orien- ta hacia una concepción integral y participativa de la cultura organizacional que permita direccionar de forma clara los esfuerzos organizativos (Deshpandé \& Farley, 2004). De este modo, la innovación constituye un componente fundamental de la estrategia organizacional, por lo que deben estable- 
cerse mecanismos de seguimiento a los procesos con el objeto de garantizar la mejora continua y la generación de valor agregado en los procesos de la organización.

El segundo componente del modelo corresponde al desarrollo de las competencias claves de innovación en el personal, específicamente relacionadas con el compromiso, la comunicación, la colaboración inteligente entre los distintos departamentos funcionales de la empresa y el sentido de pertenencia en la organización. El desarrollo de estas competencias permite superar las barreras internas que surgen frente a los procesos de cambio (De Brentani \& Ragot, 1996) y favorecen la implementación de culturas organizacionales innovadoras (Naranjo-Valencia \& Calderón-Hernández, 2015).

El tercer componente corresponde a la retroalimentación con los clientes, direccionado al fortalecimiento de los mecanismos de interacción con los mismos, considerando que representan una importante fuente de información para el sistema de innovación. Es relevante favorecer la participación del cliente en procesos de innovación (Hennestad, 1999), considerando los beneficios en términos de calidad del producto, eficiencia de los procesos y posicionamiento en el mercado de los bienes y servicios innovadores.

Adicionalmente, el desarrollo de las competencias claves de innovación en los miembros de la empresa y el desarrollo de mecanismos para la retroalimentación con los clientes genera el desarrollo de una cultura co-creadora (Ballantyne et al., 2008), en la cual el conocimiento de los colaboradores y los clientes se integra al sistema de innovación organizacional.

El cuarto componente se fundamenta en la planificación y documentación de las estrategias de innovación. Producto de este ejercicio, es fundamental definir el foco de la innovación de la organización hacia algún tipo en específico y se establecen las acciones requeridas para su desarrollo.
El Manual de Oslo de la OECD y Eurostat (2005) establece cuatro tipos de innovación: de producto, de proceso, de mercadotecnia y de organización. En este componente, la empresa objeto de estudio deben orientarse al desarrollo de innovaciones en el proceso de producción y distribución en el ámbito internacional, así como en innovaciones organizacionales, orientadas a la formas de relación de la organización con el entorno.

El quinto componente del modelo propuesto se sustenta en el seguimiento a las estrategias y a las acciones planeadas en innovación, desde un enfoque que permita identificar y potenciar oportunidades de mejora y fuentes de ventajas competitivas para la organización. Para tal efecto, es fundamental fortalecer el empoderamiento de los colaboradores y los procesos de mejora continua, así como el establecimiento de indicadores que permitan evaluar el alcance de las estrategias planteadas.

Los componentes anteriormente enunciados se integran en la dinámica empresarial mediante dos elementos bases del modelo propuesto. El primero de ellos corresponde a la adopción de la cultura de cambio en la organización y la interacción con el ámbito externo, lo cual se asocia con la mejora en los resultados empresariales (Griffin, 1997), (Hulte et al., 2004), (Menguc \& Auh, 2006). El segundo, se relaciona con la interacción de la empresa con el ambiente externo, ámbito en el cual, en la implementación de una cultura de innovación es fundamental conjugar cualidades personales, recursos organizacionales, y elementos provenientes de la interacción con el entorno de la empresa (Rodríguez et al., 2011).

El modelo propuesto también se soporta en la aplicación de los componentes del modelo de gestión de la innovación planteado por la Fundación COTEC (2004); garantizando la apropiación de una cultura efectiva de innovación en la empresa. El primer aspecto del modelo de gestión de la innovación en referencia corresponde a focalizar, el cual se asocia con el componente de articula- 
ción de la innovación a la estrategia competitiva. El segundo aspecto corresponde a la capacitación para la gestión de la innovación, asociados con los componentes de desarrollo de competencias en el personal y retroalimentación con los clientes. El tercer aspecto del modelo de gestión de la innovación es implementar, mediante la planificación y documentación de estrategias de innovación. El cuarto aspecto para gestionar la innovación es vigilar, abordado en el componente de seguimiento a las estrategias y acciones. Finalmente, el modelo de gestión de innovación contempla el aspecto de aprendizaje como transversal al desarrollo de una cultura organizacional innovadora.

Al aplicar el modelo aquí propuesto en Asoprocasines, se evidencia la necesidad de fortalecer la articulación de la innovación a la estrategia competitiva, debido a que existen falencias en la implementación y seguimiento a los procesos estratégicos, dificultando la generación de valor agregado. Del mismo modo, la empresa analizada se categoriza dentro de una cultura de grupo y racional, aunque se evidencian elementos de las culturas de desarrollo y jerárquica, validando la interacción de los diferentes elementos que confluyen en el desarrollo de la cultura organizacional. Además, existe una cultura fuerte, un desarrollo efectivo de flujos de comunicación y relación de organización en el ámbito tanto interno como externo y un marcado sentido de pertenencia; componentes que han permitido la sostenibilidad y posicionamiento de la empresa en el mercado.

Sin embargo, el análisis de la cultura organizacional de Asoprocasines presenta elementos relevantes que actúan como restrictivos en el desarrollo de la cultura innovadora, entre ellos, el hecho de que los mecanismos de cambio no se activan de manera sistemática en la cultura interna y los ejercicios de documentación, planificación y seguimiento de acciones de innovación deben fortalecerse con el objeto de garantizar procesos innovadores efectivos y desarrollo organizacional.

\section{Conclusiones}

El desarrollo de una cultura innovadora, desde el análisis efectuado para la estructuración del modelo aquí propuesto, se concibe como un proceso cíclico, consistente en un conjunto de cinco etapas direccionadas a la generación de conductas y comportamientos para impulsar el desarrollo innovador. Entre los cinco componentes del modelo se tienen: la innovación como un elemento fundamental en la dinámica de la organización, el desarrollo de competencias claves en el personal para favorecer los procesos de innovación, la retroalimentación con el cliente para favorecer su participación en el proceso de innovación, la planificación y documentación de estrategias direccionadas hacia algún tipo de innovación que genere valor para la empresa, junto con el seguimiento de las estrategias y acciones que permitan gestionar efectivamente los aprendizajes y avances del proceso de innovación.

El modelo surgió de la revisión de literatura orientada a la identificación de las diferentes dimensiones de la cultura organizacional innovadora; luego se realizó un diagnóstico de la cultura organizacional de la Asociación de Productores Orgánicos Arhuacos y Campesinos de la Sierra Nevada de Santa Marta, Asoprocasines, como unidad de análisis, para fundamentar el modelo teórico. Como trabajo futuro se sugiere aplicar el modelo en diferentes sectores o en un mayor número de empresas, con el objeto de mejorar su practicidad y validez como herramienta dinamizadora de la cultura de innovación en el contexto organizacional.

\section{Referencias}

Alam, I. (2005). Removing the fuzziness from the fuzzy front-end of service innovations through customer interactions. Industrial Marketing Management, 35 (4), 468-480. doi:10.1016/j.indmarman.2005.04.004 
Arias, F., \& Heredia, V. (2001). Administración de recursos humanos para el alto desempeño. México: Trillas.

Asoprocasines. (2011). Historia Café Nacer. Recuperado de: http://cafesespecialesdelasierranevada. com/index.php?option=com_content\&view $=$ article\&id $=87 \&$ Itemid $=87$

Ayala, P. (2014). Cultura Organizacional e Innovación. Gestión de Personas y Tecnología, 19, 27-33. Recuperado de: http://www.revistas.usach.cl/ojs/ index.php/revistagpt/article/viewFile/1474/1369

Ballantyne, D., Varey, R., Frow, P., \& Payne, A. (2008). Service-dominant logic and value propositions: Re-examining our mental models. Otago Forum 2 :Academic Papers, 42-60. Recuperado de: http://citeseerx.ist.psu.edu/viewdoc/download?doi=10.1.1.517.3480\&rep=rep1\&type $=$ pdf

Cameron, K., \& Quinn, R. (1999). Diagnosing and changing organizational culture: based on the competing values framework. Reading, Inglaterra: Addison-Wesley.

De Brentani, U., \& Ragot, E. (1996). Developing new business-to-business professional services: what factors impact on performance. Industrial Marketing Management, 25 (6), 571-530. doi: 10.1016/ S0019-8501(96)00066-1

Deshpandé, R., \& Farley, J. (2004). Organizational culture, market orientation, innovativeness, and firm performance: an international research odyssey, organizational culture. International Journal of Research in Marketing, 21 (1), 3-22. doi:10.1016/j. ijresmar.2003.04.002

Dewitt, T., \& Brady, M. (2003). Rethinking service recovery strategies: the effect of rapport on consumer responses to service failure. Journal of Service Research, 6 (2), 193-207. doi:10.1177/1094670503257048
Escorsa C, P., \& Valls P, J. (2003). Tecnología e Innovación en la empresa. Barcelona, España: Edicions de la Universitat Politecnica de Catalunya, UPC.

Finol, \& Nava. (1999). Procesos y productos en la investigación documental. Segunda Edición. Maracaibo, Venezuela: Editorial de la Universidad del Zulia.

Fundación COTEC para la Innovación Tecnológica. (2004.). Informes sobre el sistema español de innovación: análisis del proceso de innovación en las empresas de servicios. Madrid, España: COTEC.

Future Trends Forum. (2007). Innovación: the wealth of nations, resumen ejecutivo. Madrid, España: Fundación de la Innovación.

Gibson, Ivancevich, \& Donnelly. (2002). Las organizaciones: comportamiento, estructura y procesos (10ª ed). México D.F:, México: McGraw-Hill.

Griffin, A. (1997). PDMA research on new product development practices: updating trends and benchmarking best practices. Journal of Product Innovation Management, 14 (6), 429-458. doi:10.1016/ S0737-6782(97)00061-1

Hennestad, B. (1999). Infusing the organisation with customer knowledge. Scandinavian Journal of Management, 15 (1), 17-41. doi:10.1016/S09565221(97)00050-X

Hernández S., R., Fernández C., C., \& Baptista, P. (2010). Metodología de la investigación. (5a ed). Mexico. D.F, México: Mc Graw Hill.

Hill, C., \& Jones, G. (2001). Administración estratégica, un enfoque integrado. Bogotá, Colombia: McGraw-Hill.

Hitt, M., Ireland, R., \& Hoskisson, R. (1999). Administración estratégica: competitividad y conceptos de globalización. México D.F., México: International Thompson. 
Hult, G., Hurley, R., \& Knight, G. (2004). Innovativeness: its antecedents and impact on business performance. Industrial Marketing, 33 (5), 429-438. doi:10.1016/j.indmarman.2003.08.015

Hurley, R., \& Hult, G. (1998). Innovation, market orientation and organization learning: an integration and empirical examination. Journal of Marketing, 62, 42-54. doi: 10.2307/1251742

Menguc, B., \& Auh, S. (2006). Creating a firm-level dynamic capability through capitalizing on market orientation and innovativeness. Journal of the Academy of Marketing Science, 34 (1), 63-73. doi: 10.1177/0092070305281090

Naranjo-Valencia, J. C., \& Calderón-Hernández, G. (2015). Construyendo una cultura de innovación. Una propuesta de transformación cultural. Estudios Gerenciales, 31, 223-236. doi: 10.1016/j.estger.2014.12.005

Naranjo-Valencia, J. C., Jiménez, D., \& Sanz-Valle, R. (2012). ¿Es la cultura organizativa un determinante de la innovación en la empresa?. Cuadernos de Economía y Dirección de la Empresa, 15 (2), 63-72. doi:10.1016/j.cede.2011.07.004

Organización para la Cooperación y el Desarrollo Económico OCDE. (2003). Manual de Frascati: propuesta de norma práctica para encuestas de investigación y desarrollo experimental. París, Francia: Fundación Española Ciencia y Tecnología FECYT.

Organización para la Cooperación y el Desarrollo Económico, OECD; Oficina de Estadísticas de las Comunidades Europeas, Eurostat. (2005). Manual de Oslo, guía para la recogida e interpretación de datos sobre innovación. (3a. ed). España: Grupo Tragsa.

Ottenbacher, M., Gnoth, J., \& Jones, P. (2006). Identifying determinants of success in development of new high-contact services. International Journal of Service Industry Management, 17 (4), 344-363. doi:10.1108/09564230610680659

Páramo, D. (2001). Hacia la construcción de un modelo de cultura organizacional orientada al mercado. Revista Colombiana de Marketing, 2 (2), 1-26. Recuperado de: http://www.redalyc.org/articulo. oa?id=10900206

Pavón, J., \& Goodman, R. (1981). Proyecto MODELTEC, la planificación del desarrollo tecnológico. Madrid, España: CDTI-CSIC.

Pirela, A., Rengifo, R., Arvanitis, A., \& Mercado, A. (1991). Conducta empresarial y cultura tecnológica: empresas y Centros de Investigación en Venezuela. Caracas. Venezuela: Cendes.

Ramos-Parra, C. (2006). Cultura innovativa en la vinculación de las universidades públicas del Zulia con el entorno socioproductivo (Tesis de maestría). Universidad Rafael Belloso Chacín, Venezuela. Recuperado de http://tesis.luz.edu.ve/tde_busca/arquivo.php?codArquivo $=1259$

Ramos-Parra, C. (2012). Cultura innovativa para la universidad de servicio en el marco de la responsabilidad social universitaria. Opción, 28 (68), 287-302. Recuperado de: http://www.redalyc.org/ pdf/310/31025437003.pdf

Rodríguez, A., Araujo, A., Hagemeister, M., Landeta, J., Larrauri, M., \& Ranguelov, S. (2006). Capacidad empresarial para la absorción de resultados de I+D: un enfoque de conocimiento, principios, análisis empíricos y directrices de autoevaluación. Bilbao, España: Servicio Editorial de la Universidad del País Vasco.

Rodríguez, A., Hoyos I., J., Izaguirre O, J., \& Vicente M. A., (2011). Organizaciones en el marco de una cultura social innovadora: propuesta de factores explicativos. Investigaciones Europeas de Dirección y Economía de la Empresa, 17 (1), 17-35. Recupera- 
do de: http://www.aedem-virtual.com/articulos/ iedee/v17/171017.pdf

Schein, E. (1992). Organizational culture and leadership. (2a ed). San Francisco, Estados Unidos: Jossey-Bass.

Schumpeter, J. (1934). The theory of economic development. Cambridge, Estados Unidos: Harvard University Press.

Sundbo, J. (1997). Management of innovation in services. The Service Industries Journal, 17 (3),
432-455. Recuperado de: http://people.ischool. berkeley.edu/ glushko/ISE-Notes-Fall2006/Sundbo-ServicesInnovation.pdf

Sundbo, J., \& Gallouj, F. (2000). Innovation as a loosely coupled system in services. International Journal of Services Technology and Management, 1, 43-68. doi: 10.1504/IJSTM.2000.001565

Tamayo, \& Tamayo, M. (2011). El proceso de la investigación científica. ( $5^{\mathrm{a}}$ ed). México D.F., México: Limusa S.A. 